\title{
CHANDLER EFFECT AND NEARLY DIURNAL FREE WOBBLE AS DETERMINED FROM OBSERVATIONS WITH A SUPERCONDUCTING GRAVIMETER
}

\author{
Bernd Richter \\ Institut für Angewandte Geodäsie \\ Richard-Strauss-Allee 11 D-6000 Frankfurt 70, FRG \\ Walter Zürn \\ Geowissenschaftliches Observatorium Schiltach \\ Heubach 206 D-7620 Wolfach, FRG
}

\begin{abstract}
A continuous three year series of observations with the model TT40 superconducting gravimeter has been analysed for all frequency domains. The frequency of the nearly diurnal free wobble (NDFW) $(1+1 /(431.2 \pm 3.2)$ cycles/sid.d.) and a damping factor (6208.5 days) are derived from this dataset. These results are not in agreement with the values predicted by the theoretical model presented by Wahr but match very closely the values derived from VLBI observations.

A similar study of long period effects suggests furthermore that there is a strog correlation with the polar motion observed by other techniques. By comparing different drift models with respect to the ultra-long period of the gravity variations it is shown that the observed Chandler effect is not principally subjected to this type of instrumentally dependent parameter.
\end{abstract}

\section{Introduction}

The analysis of precise earth tide observations is one tool to get informations on the elastic behaviour of the earth in different frequency domains. The major waves of the tidal spectrum appear in the diurnal and semidiurnal band whereas long period tidal variations ( $<14$ days) are small and latitude dependent. For the earthtide observation station established by the Institut für Angewandte Geodäsie, at the castle in Bad Homburg $\left(\Phi=50.2285^{\circ} N, \lambda=8.6113^{\circ} E, h=190 m\right)$ the long period portion of the tidal spectrum is less than $5 \%$ of the total gravity signal. As a result of the long-term stability of the superconducting gravimeter (SCG) the whole tidal spectrum as well as changes in the centrifugal force due to the polar motion can be observed for the first time.

In this report the data of a three year uninterrupted registration (May 1981-April 1984) with the SCG No. TT40 are investigated.

The data analysis is performed by the HYCON-method, which allows a simultaneous solution of tidal parameters, instrumental drift and the determination of regression factors for the different input channels (Schüller, 1986).

2. Properties of the nearly diurnal free wobble derived from diurnal gravity tides.

A well known method to determine the complex frequency response of a linear physical system in the laboratory is, to subject the system successively to a series of harmonic driving forces with measured frequencies and amplitudes and to compare amplitudes and phases ot the output and input signals. If the system has resonances (eigenmodes) in the frequency band used, these will 
input signals. If the system has resonances (eigenmodes) in the frequency band used, these will show up in the response. An important point in the laboratory is to wait until the transients from switching on the driving force have died out and a steady state is reached. Note that these transients correspond to excitations of the eigenmodes which for a stable system will decay i.e. in the stady state the eigenmodes are not excited unless the driving frequency equals the eigenfrequency. Nature has set up such an experiment on a grand scale. The (almost) harmonic tidal forces in the diurnal frequency band test the earth in the vicinity of an eigenmode, the "nearly diurnal free wobble" (NDFW). There are however differences to the experiment in the laboratory: all frequencies are applied simultaneously, they are not nicely spaced in the frequency domain and the amplitudes of the different constituents vary by orders of magnitude; furthermore, the tide closest to the NDFW-eigenfrequency, $\psi_{1}$, is unfortunately very small. Whereas many investigations of the diurnal tides using gravity, tilt or strain observations were carried out to show that the theoretically predicted resonance exists (e.g. Melchior, 1983; Leoine, 1978) very few attempts have been made to extract new information on the resonance from tidal measurements (Warburton $\mathcal{E}$ Goodkind, 1978; Goodkind, 1983; Lecolazet, 1983; Neuberg \& Zürn, 1986).

The record from the superconducting gravimeter at Bad Homburg has three credits for such an investigation:

1. The length of 33 months allows resolution of the tides $P_{1}, S_{1}, K_{1}, \psi_{1}, \phi_{1}$.

2. It is probably the tidal record with the lowest noise level in the diurnal band get obtained, even taking into account that only about $16 \%$ of the signal have geophysical meaning in the gravity tides it is a very good signal-to-noise ratio.

3. The contributions of the diurnal ocean tides to the gravimetric tides in Central Europe are very small, so errors in the corresponding corrections have a small effect as well.

The diurnal gravimetric admittances (i.e. gravimetric factors $\delta_{K}$ and phase leads $\kappa_{K}$ ) were obtained using Schüller's (1986) method (HYCON-MC), with local barometric pressure being the only additional input signal besides the theoretical tides. The formal uncertainties $\sigma_{K}$ at these least squares estimates were later used as weights in the analysis of the resonance (see table 1). The gravity signals at Bad Homburg from the ocean tides were computed from Schwiderski's models (DUCARME, pers. comm.). These ocean contributions were applied as phasor corrections to the observed complex admittances after normalisation to the rigid earth tides. Since ocean models for $\psi_{1}$ and $\phi_{1}$ are not available, different schemes were used by $Z u ̈ r n$ et. al. (1986) with negligible effects on our results. The resonance contribution (NDFW) is separated from the static response by subtracting the complex admittance $\delta \cdot \exp (i \kappa)(i=\sqrt{-1})$ of $O_{1}$ from those of the four other tides. The latter procedure also reduces the influence of calibration uncertainties on the resonance structure. The complex resonance contributions, also listed in table 1 , are used for this method together with the $\sigma_{K}$ as input data to find the parameters of the eigenmode. As a model for the resonance structure the frequency dependence of the Love numbers due to the NDFW as given by $W a h r(1981)$ is taken and attenuation is introduced as a small perturbation by replacing $\omega_{0}$ by $\omega_{0}(1+i / 2 Q) . Q$ represents the quality factor of the NDFW-eigenmode and $\omega_{0}$ its eigenfrequency. For the difference in admittances of tide $k$ and $O_{1}$ we get (Zürn et al., 1986):

$$
R\left(\omega_{k}\right)=\delta\left(\omega_{k}\right)-\delta\left(\omega_{O_{1}}\right)=\frac{A\left(\omega_{k}-\omega_{O_{1}}\right)}{\omega_{0}-\omega_{k}+i \omega_{0} / 2 Q}
$$

where $\omega_{k}$ is the frequency of tide $k . A$ is the dimensionless complex strength of the resonance, a physical property of the earth ( see Neuberg et al., 1987) and it must not be confused with the excitation amplitude of the NDFW, which is not important for this purpose and cannot be determined with this method. $Q$ is the number of oscillations of the mode after which the amplitude would drop by the factor of $\exp (-\pi)$ if excited and left alone. The model, eq. (1) is used in a weighted non-linear least squares fit to the complex resonance contributions derived from the observations 


\begin{tabular}{|l|r|r|r|r|r|}
\hline tide: & $\mathrm{O} 1$ & $\mathrm{P} 1$ & $\mathrm{~K} 1$ & $\psi_{1}$ & $\phi_{1}$ \\
\hline HYCON- & & & & & \\
results & & & & & \\
$\delta:$ & 1.1449 & 1.1455 & 1.1331 & 1.2427 & 1.1689 \\
$\kappa:$ & -0.07 & 0.06 & 0.10 & 1.57 & -0.16 \\
$\sigma:$ & 0.0001 & 0.0003 & 0.0001 & 0.0116 & 0.0065 \\
\hline oceanic & & & & & \\
loading & & & & & \\
(DUCARME) & & & & & \\
amplitude: & 0.143 & 0.056 & 0.172 & - & - \\
phase lead: & 164.33 & 72.56 & 59.17 & - & - \\
\hline resonance eff. & & & & & \\
real: & 0.00 & -0.0051 & -0.0185 & 0.0928 & 0.0195 \\
imaginary : & 0.00 & 0.0001 & 0.0012 & 0.0367 & -0.0006 \\
\hline
\end{tabular}

Tabelle 1: Gravimetric factors $\delta$, phase leads $\kappa$ (in degrees) and formal uncertainties $\sigma$ (of $\delta$ ) as found by HYCON-MC; ocean load corrections as computed by Ducarme from Schwiderski's models (amplitude in $10^{-8} \mathrm{~ms}^{-2}$, phase leads in degrees) and resonance contributions for five diurnal tides.

(8 equations, 4 unknowns: $\omega_{0}, Q, A=A_{R}+i A_{I}$ ).

The results are:

$$
\begin{aligned}
\omega_{0} / 2 \pi & =1.005066 \pm 0.000017 c p d \\
& =15.075952 \pm 0.000225^{\circ} / h r \\
& =1+1 /(431.2 \pm 3.2) c p \text { sid.d. } \\
Q & =3120 \pm 323 \\
A & =-(0.00059+i \cdot 0.000002)
\end{aligned}
$$

The free core nutation (FCN) associated with the NDFW has a period of 431.2 siderial days. The recent estimate from VLBI is 432.7 sid. days (Gwinn et al., 1986), so experimental agreement for the eigenfrequencies between gravity tides and VLBI is excellent (see also Neuberg et al., 1987. Wahr's (1981) earth models provide FCN periods close to 460 sid. days. Some recent estimates of the effects of mantle anelasticity (Wahr\&Bergen, 1986; Dehant, 1987) increase the discrepancy between theory and observation. Possible causes are discussed by Gwinn et al. (1986) and Neuberg et al. (1987), the most likely being an increase of the ellipticity of the core-mantle boundary from the hydrostatic values assumed in the theories adopted so far. Before theory can explain this discrepancy, a geophysical interpretation of the strength $A$ cannot be attempted with confidence. Since the NDFW and the FCN are only two aspects of the same mode, their amplitudes are strongly related (Toomre, 1966) and therefore they must decay with the same decay time constant $\tau=I m^{-1}\left(\omega_{0}\right)$. Our $Q$ estimate leads to $\tau=2 Q / \omega_{0}=988$ days $(2.71$ years $)$ while the VLBI estimate of Gwinn et al. (1986) is $\tau=6901$ days. The uncertainties on both estimates are $10 \%$ and $50 \%$ respectively, so at the one standard deviation level they disagree. This discrepancy can only be solved by better observations. WahrEBergen (1986) have estimated a decay time $\tau$ from 20000 to 124000 days due to mantle anelasticity alone. Every other mechanism will lower the decay time, one possibilty being the damping due to the ocean (Goodkind, 1983), while magnetic and viscous coupling at the core-mantle boundary are considered to contribute very little to the damping (Hinderer, 1986).

\section{Polar motion observed by gravity}

The long term stability of the SCG offers the possibility to study the long period gravity variations. 
Nevertheless the instrument has a drift of $\sim 50 \cdot 10^{-8} \mathrm{~ms}^{-2} / 3$ years which is due to a very small leak in the instrument. To eliminate the drift, different driftmodels on the long period parameters are fitted in a least square procedure (Richter, 1986). The result is given in table 2.

\begin{tabular}{|c|c|c|c|c|c|c|c|}
\hline drift model & \multicolumn{2}{|c|}{ Chandler wobble } & \multicolumn{2}{|c|}{ Sa } & \multicolumn{2}{|c|}{ Ssa } & $\sigma^{2}$ \\
& ampl. & deg. & ampl. & deg. & ampl. & deg. & \\
\hline & & & & & & & \\
$a+b x+c x^{2}$ & 4.43 & -172.4 & 1.41 & 43.8 & 2.31 & 18.4 & 0.166 \\
$a+b x+c x^{2}+d x^{3}$ & 4.40 & -170.4 & 1.29 & 48.3 & 2.30 & 17.9 & 0.164 \\
$a+b e^{-c t}$ & 4.42 & -159.6 & 1.04 & 88.0 & 2.29 & 15.7 & 0.239 \\
$a+b \cos \left(\frac{2 \pi}{9 a}+\phi\right)$ & 4.68 & -174.6 & 2.25 & 24.3 & 2.34 & 21.0 & 0.313 \\
& & & & & & & \\
\hline
\end{tabular}

Tabelle 2: different drift models (quadratic, cubic, e-function, cos-function (9 years); (ampl. = amplitude in $\left.10^{-8} \mathrm{~ms}^{-2}\right)$ )

It is mainly the phase relation which is influenced by the different drift models. The remainining analysis uses the cubic polynomial drift model which gives the best fit.

The change of the centrifugal force is due to the change in the position of the rotational axis of the earth which leads to a latitude variation of the station. The mathematical background has been derived by several authors (Heitz, 1980/83; Wahr, 1985; Lambeck, 1973) so that here only the final equation is cited:

$$
\begin{gathered}
b_{3 . z}^{a} \quad\left(\partial W_{R} / \partial \bar{r}\right)=-\frac{2 \delta}{\bar{r}} W_{R} \\
\approx-\delta \bar{\omega}^{2} \bar{r} \sin \Phi\left(n_{1} \cos \lambda+n_{2} \sin \lambda\right)
\end{gathered}
$$

with $W_{R}$ denoting the rotational potential, $\bar{r}$ the earth radius, $\delta$ the gravimetric factor, $\bar{\omega}$ the earth rotational speed and $n_{1}, n_{2}$ the pole coordinates in rad.

To determine a possible phase lag equation (2) is rewritten as:

$$
b_{3 . z}^{a}=n_{1} k_{0} \delta \cos \lambda+n_{2} k_{0} \delta \sin \lambda=n_{1} U_{1}+n_{2} U_{2}
$$

with $U_{1}=k_{0} \delta \cos \lambda ; U_{2}=k_{0} \delta \sin \lambda$ and $k_{0}=-3.36 \cdot 10^{6} \sin 2 \Phi$.

The positions of the rotational axis (pole position) are published by the Bureau International d'Heure and the U.S. Naval Observatory for various intervals of time. Due to the fact that the functional variation of the centrifugal force can hardly be described by harmonic variations the interpolated hourly values resulting from this influence are added to the model as additional input channels. Two different input sets have been used for the polar coordinates: 1 . smoothed BIH data, 2. raw IRIS observations. The results for the only two 'polar cycles' observed by both celestrial and gravitational means are:

for the smoothed BIH data

$$
\delta=1.52 \pm 0.22 \text { and } \lambda=8.0^{\circ} \pm 5.1^{\circ} ;
$$

for the raw IRIS data

$$
\delta=1.27 \pm 0.16 \text { and } \lambda=7.7^{\circ} \pm 3.2^{\circ} .
$$


The results derived with the IRIS data set fit better to the gravity observation and the residuals after this fit are $30 \%$ smaller when compared with the residuals from the smoothed BIH data as input channel.

The residuals of the analysis, where a complete earth tide model, the air pressure and the IRIS polar motion data are taken into account, are demonstrated in figure 1. For the most part the residuals are in the range less than $10^{-8} \mathrm{~ms}^{-2}$ and are caused by an incomplete air pressure model (only the local air pressure is taken into account), disregarding solar radiation, groundwater influences as well as instrumental disturbances.

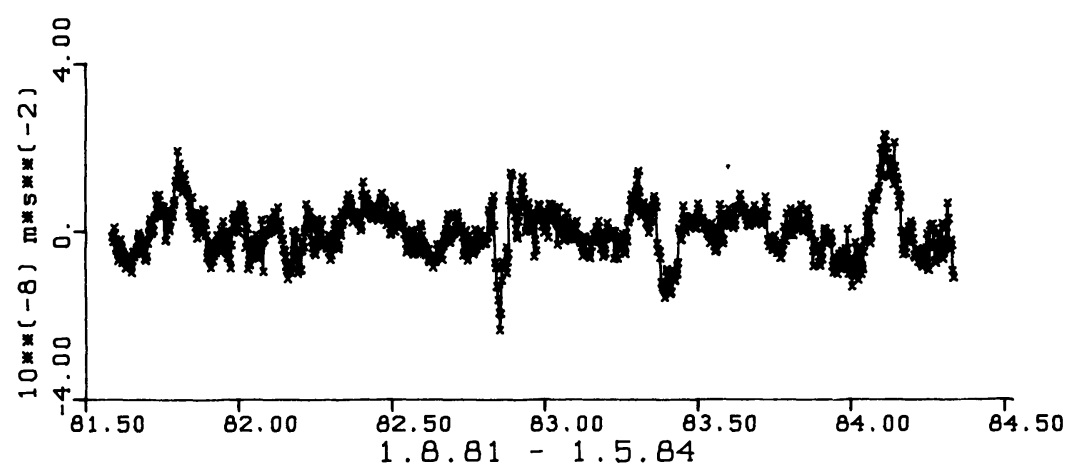

Abbildung 1: Residuals of a complete earth tide analysis plus the air pressure and the polar motion as additional input parameter for the least squares adjustment

This demonstrates that the gravity data are sensitive to the short period variations in the polar motion, but the adjusted $\delta$-factor is more than $10 \%$ higher than that anticipated theoretically $\left(\delta_{\text {theo }}=1.16\right)$, whereas no significant phase lag between celestial and gravitational data can be observed $\left(\lambda_{B I H, I R I S}=\lambda_{S T A T I O N}\right)$.

Two explanations for this discrepancy can be offered; first the fit is performed in the time domain (there could be frequency dependent parameters of the long period elastic behaviour of the earth which are not taken into account) and second the polar motion has also a strong annual portion which could match with the annual period of the tides in the analysis procedure. Both of these could therefore lead to an unrealistic $\delta$-factor. To overcome these problems a solution is being developed that describes only the earth response to the Chandler wobble frequency.

\section{Acknowledgement}

Discussions with J. Neuberg, J. Hinderer and P. Rydelek are appreciated. DFG supported parts of this reseach under grant $\mathrm{Wi} / 687$. 


\section{References}

Dehant, V. ,1986: 'Nutations and Inelasticity of the Earth.' submitted to Physics of the Earth and Planetary Interiors

Goodkind, J. ,1983: 'Q of the nearly diurnal free wobble.' Proc. 9th Int. Symp. Earth Tides (ed. J. Kuo), Schweizerbart, Stuttgart, 569-575

Gwinn, C. R.; Herring, T. A.; Shapiro, I. I., 1986: 'Geodesy by Radio Interferometry: Studies of the forced nutation of the earth. 2. Interpretation. ' J. Geophys. Res.91, 4755-4765

Heitz, S., 1980/83: 'Grundlagen kinematischer und dynamischer Modelle der Geodäsie, Geophysik und Astronomie, Band 1 und 2.' Ferd. Dümmler's Verlag, Bonn

Hinderer, J.; Legros, H.; Amalvict, M.; Lecolazet, R., 1986: 'Theoretical and Observational Search for thr Gravimetric Factor: Diurnal Resonance and Long-period Pertubations.' Proc. 10th Int. Symp. Earth Tides (ed. Vieira), Madrid, in press

Lambeck, K., 1973: 'Temporal variations of rotational origin in the absolute value of gravity.' Studia geophys. et geod.17, 269-271

Lecolazet, R., 1983: 'Correlation between diurnal gravity tides and the earth's rotation rate.' Proc. 9th Int. Symp. Earth Tides (ed. Kuo), Schweizerbart, Stuttgart, 527-530

Leoine, J., 1978: 'Strain-Tide Spectroscopy.' Geophys. J. R. astro. Soc.54, 27-41

Melchior, P., 1983: 'The Tides of the Planet Earth, 2nd edition.' Pergamon Press, Oxford

Neuberg, J.; Hinderer, J.; Zürn, W., 1987: 'Stacking gravity tide observations in Central Europe for the retrieval of the complex eigenfrequency of the nearly diurnal free wobble.' submitted to Geophys. $J$. R. astr. Soc.

Neuberg, J.; Zürn, W., 1986 : 'Investigation of the nearly diurnal resonance using gravity, tilt and strain data simultaneously.' Proc. 10th Int. Symp. Earth Tides (ed. Vieira), Madrid, in press

Richter, B., 1986: 'The spectrum of a registration with a superconducting gravimeter.'Proc. 10th Int. Symp. Earth Tides, (ed. Vieira), Madrid, in press

Schüller, K., 1986: 'Simultaneous tidal and multi-channel input analysis as implemented in the HYCON method.' Proc. 10th Int. Symp. Earth Tides, (ed. Vieira), Madrid, in press

Toomre, A., 1966: 'On the "nearly diurnal free wobble" of the earth.' Geophys. J. R. astr. Soc.38, 335-348

Wahr, J. M., 1981: 'Body tides of an elliptical, rotating elastic and oceanless earth.' Geophys. J. R. astr. Soc.64, 677-703

Wahr, J. M., 1985: 'Deformation induced by polar motion.'J. Geophys. Res. 90, 9363-9368

Wahr, J. M.; Bergen, Z., 1986: 'The effects of mantle anelasticity on nutations, earth tides, and tidal variations in rotation rate.' Geophys. J. R. atsr. Soc.87, 633-668

Warburton, R. J.; Goodkind, J. M., 1978: 'Detailed gravity-tide spectrum between one and four cycles per day.' Geophys. J. R. astr. Soc. 52, 117-136

Zürn, W.; Rydelek, P. A.; Richter, B., 1986: 'The core-resonance effect in the record from the superconducting gravimeter at Bad Homburg.' Proc. 10th Int. Symp. Earth Tides (ed. Vieira), Madrid, in press 


\section{DISCUSSION}

Groten: If you actually fit your gravimetric data to observed VLBI data, i.e. to the observed uplift and/or subsidence, then you have the Love number $h$ inherent in the VLBI data and you are not actually using a rigid Earth model with $h=0$.

Reply by Richter: Only the VLBI derived polar coordinates are used in predicting the varying centrifugal force for a rigid Earth model.

Pâquet: Do you have a physical interpretation of the $\delta$ factor value (1.5) that you obtain? This factor is expected to be equal to 1.16 .

Reply by Richter: The $\delta$ factor we calculate is predicted in the time domain and therefore not directly comparable with the $\delta$ factor in the frequency domain, except the $\delta$ factor in the frequency domain is constant (1.16) for all frequencies wh ich are involved in the polar motion.

Yoder: 1) Have you attempted to analyze $\delta$ at annual and Chandler wobble frequencies? 2) What is the contribution of seasonal air pressure corrections to effective $\delta$ factor?

Reply by Richter: With the three year data set, it is not possible to determine the $\delta$-factor for the annual and Chandler period by spectral methods. The polar motion is correlated in the time domain where also the non-periodic components can be compared. Therefore, the derived $\delta$-factor is an overall one and does not represent the relation for a special frequency. The data are reduced by the local air pressure effects in the time domain, so that at the moment no special spectral information can be given.

Dehant: When you take mantle inelasticity into account, you can increase the gravimetric factor by about $10 \%$ with respect to the elastic value. But I think you will never get such a high value of 1.5 when you compute the effect of the pole tide. 